\title{
An Avida-ED digital evolution curriculum for undergraduate biology
}

\author{
James J. Smith 1,2,3,4,5*, Wendy R. Johnson ${ }^{5,6}$, Amy M. Lark ${ }^{7}$, Louise S. Mead ${ }^{3,4,5}$, Michael J. Wiser ${ }^{4,5}$ \\ and Robert T. Pennock ${ }^{1,4,5,8,9}$
}

\begin{abstract}
We present an inquiry-based curriculum based on the digital evolution platform Avida-ED (http://avida-ed.msu.edu). We designed an instructional sequence and lab book consisting of an introduction to Avida-ED and a set of three lessons focused on specific evolutionary concepts. These served to familiarize students with experimental evolution and Avida-ED. Students then developed independent Avida-ED research projects to test their own questions. Curriculum design and implementation occurred over the course or two semesters, with a pilot implementation in the first semester, followed by curriculum revision and full implementation in the second semester. The curriculum was implemented in an undergraduate Introductory Cell and Molecular Biology course at a major research university. Full implementation of the curriculum in semester two involved the use of Avida-ED mainly in the teaching lab in parallel with a bacterial antibiotic resistance experimental research stream, allowing students to draw connections between Avidian digital evolution and the evolution of antibiotic resistance in microbial populations. After carrying out the introductory exercises, students developed independent Avida-ED projects to test their own research questions, and presented their data to researchers in the NSF-funded BEACON Center for the Study of Evolution in Action. Preliminary results of our studies to assess the impacts of an Avida-ED curriculum indicate a positive effect on student learning of evolutionary concepts, particularly in increasing the level of complexity of student explanations about the random nature of mutation.
\end{abstract}

Keywords: Evolution education, Artificial life, Avida, Avida-ED, Digital evolution, Introductory biology

\section{Background}

Evolution presents a challenging set of concepts and principles for students to master, with these challenges amplified due to societal resistance (Nelson 2012). Many misconceptions about evolutionary principles have been identified (Alters and Nelson 2002; Gregory 2009), and many resources are available to teachers as they find ways to help students understand evolutionary concepts (e.g., Understanding Evolution website; Goldsmith 2003; Abraham et al. 2009; White et al. 2013). Despite this abundance of teaching resources, teaching evolution through an inquiry approach remains somewhat intractable due to the timeframes typically involved in evolutionary

\footnotetext{
*Correspondence: jimsmith@msu.edu

${ }^{1}$ Michigan State University, Lyman Briggs College, 919 E. Shaw Lane, Room E35, East Lansing, Ml 48825-3804, USA

Full list of author information is available at the end of the article
}

processes and other difficulties of trying to study evolution in the laboratory classroom.

Speth et al. (2009) described the use of digital organisms and the digital evolution platform Avida-ED (http:// avida-ed.msu.edu) as a potential way to overcome some of the difficulties in teaching the dynamic aspects of evolutionary processes. In this paper, we extend the previous work by presenting an inquiry-based curriculum, based on Avida-ED, that addresses specific evolution misconceptions held by students. In addition, the curriculum provides a framework for working with students to design and carry out evolution experiments.

\section{What is Avida-ED?}

The research tool Avida is an artificial life platform that was developed at Cal Tech in the late 1990s, with continued development at Michigan State University (Ofria and Wilke 2004). The name, "Avida", is a Spanish abbreviation 
of artificial life, or "A-life". Many researchers have used Avida to explore fundamental questions about evolutionary processes, including questions that can be difficult to address using biological organisms (e.g., Lenski et al. 2003; Ofria et al. 2003; Misevic et al. 2006; Elena and Sanjuan 2008; Clune et al. 2011; Zaman et al. 2014).

Avida-ED is an adaptation of Avida that turned the research platform into an education tool (Pennock 2007a). The main objective of this adaptation was to give students an evolutionary lab bench that produces research-grade data, allowing them to learn not only basic evolutionary principles but also the nature and practices of science. Avida-ED is particularly well suited for dynamic, visual representations of evolutionary and scientific processes, providing an easy way for students to carry out experiments in evolution, without the need for laboratory space to do so (Speth et al. 2009).

\section{How does Avida-ED work?}

An Avidian is a computer program consisting (in the base case) of a set of 50 computer commands arranged in a loop, which comprise its "genome". The Avidian "organism" used in Avida-ED as the ancestor is programmed to make a copy of itself when the program is run (Fig. 1a). In the absence of mutation, Avidians will make perfect copies of themselves. However, when mutations are introduced, Avidians make imperfect copies of themselves at some given rate. Thus, when the ancestor is used to begin a population of Avidians in the virtual Petri dish, and a mutation rate is set, random variation is introduced into the resulting Avidian population during replication (Fig. 1b).

Avidians have a "virtual metabolism" that allows them to gain extra "energy" (processing power) from resources in their environment if they evolve the ability to process those resources. Details of the system have been described elsewhere (Ofria and Wilke 2004), but the basic idea is that through random substitution mutations Avidians evolve the ability to perform Boolean logic operations on numbers they receive as inputs from the environment. Simple operations provide a small energy boost; difficult operations give a larger boost.

Some of these resulting Avidians will have faster metabolic rates (they use data faster) and some of them will take less time to make copies of themselves (they have a shorter gestation time ${ }^{1}$ ). In either case, they will produce more offspring per unit time and are thus more fit in the biological sense. What is important to recognize about this model is that it is not a simulation, but an instantiation of the causal elements of the evolutionary

\footnotetext{
1 "Gestation time" will be referred to as "generation length" in future iterations of Avida and Avida-ED.
}

mechanism (Pennock 2007b). New variations of organisms arise through random changes in organisms' genomes; these changes are heritable; and they are the source of differential functionality, leading to natural selection as organisms compete for space in the virtual Petri dish (Fig. 1b). The organisms are not preprogrammed with regard to how they adapt to their environment and they are not rewarded by how they accomplish a task, but just by the results-evolve to perform a rewarded function and the resulting boost lets one replicate faster, thus contributing more offspring to the next generation than other organisms that are not as competitive in that environment. Avida-ED's graphical representations of populations of organisms, their genomes, and their operations allows students to observe evolution in action and run their own experiments to test evolutionary hypotheses.

\section{Avida-ED curriculum development process}

We developed and pilot-tested an Avida-ED curriculum over two semesters in spring semester 2014 in an Introductory Cell and Molecular Biology course (LB145) followed by full implementation in that same course in fall 2014. We took advantage of the summer months to evaluate the results of the spring 2014 implementation and modify the curriculum accordingly, as a part of our teaching and learning cycle (Elwood and Klenowski 2002).

Spring 2014 represented the formative phase of the Avida-ED curriculum, with implementation primarily in the lecture section of LB145. Table 1 shows the timeline of implementation of Avida-ED in this course. The curriculum in spring 2014 consisted of mini-lectures, demonstrations of and practice with Avida-ED in class, and assignments that were handed out to students in class the day that activities occurred. In spring 2014 we took advantage of a parallel section of LB145 that was being taught by a different instructor, using essentially the same curriculum and instructional model, but without using Avida-ED. This pairing provided us with a quasicontrolled experimental framework within which we collected data pertaining to student knowledge of various evolutionary concepts.

We reviewed and improved the spring 2014 implementation over the summer. Among the major changes made was pairing Avida-ED with a wet lab curriculum in which student teams isolated antibiotic resistant bacteria from different soil environments and characterized them in the teaching lab (Luckie et al. 2013). This change was motivated by Johnson's (2012) study showing that pairing Avida-ED with a wet lab in a high school AP Biology course appeared to strengthen students' abilities to draw connections between the Avidians (digital organisms) 


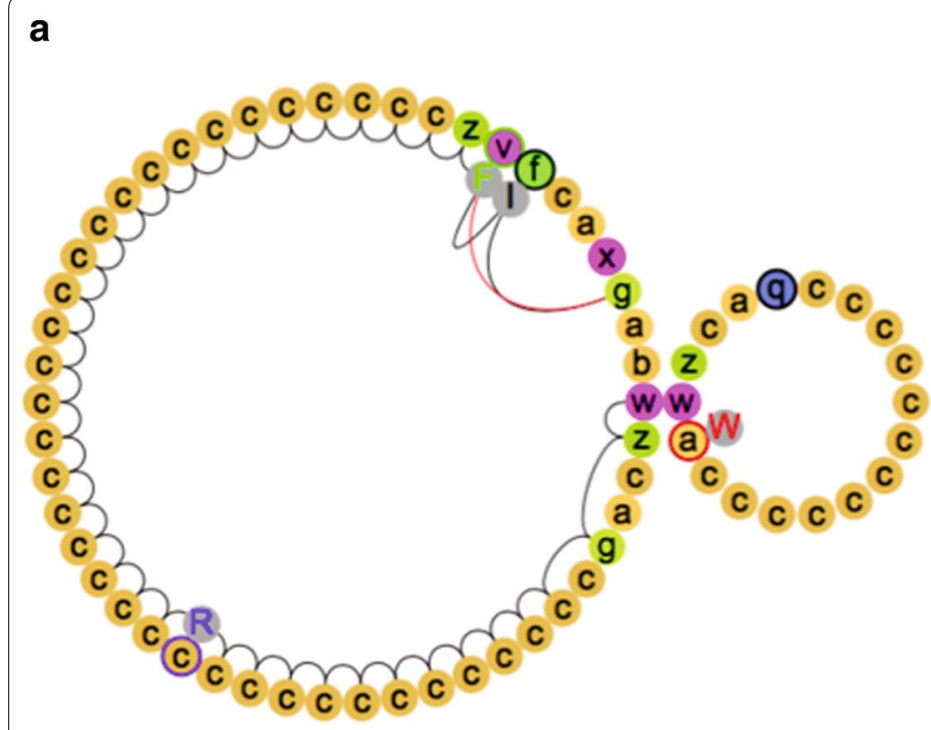

b

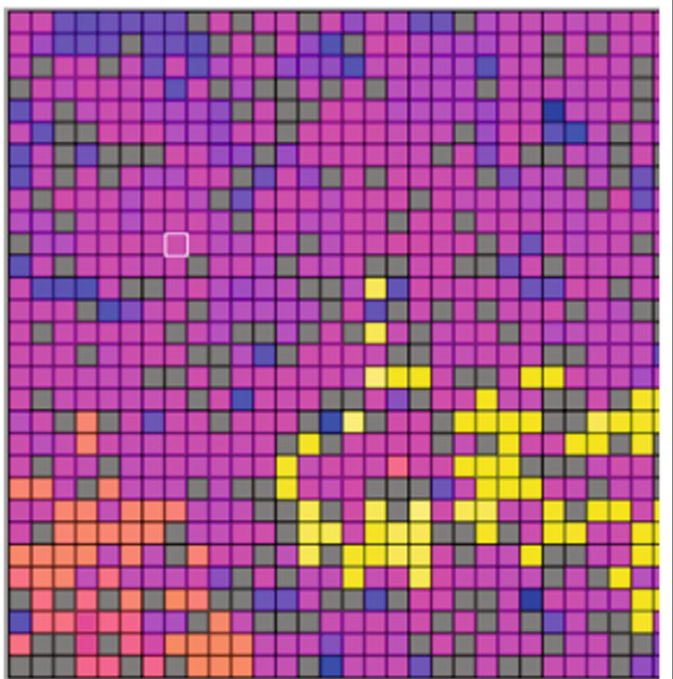

Fig. 1 Representative Avida-ED individual and population. a The circular genome of a replicating Avidian individual. Each letter in the circular sequence represents an individual command from the set of 26 commands. Each command may randomly mutate to some other command during replication (e.g., the purple $q$ in the incomplete offspring genome) and new sequences of commands may produce rewardable functions. b The Avida-ED virtual Petri dish shows a population of Avidians. Colors indicate properties of organisms (e.g., fitness, metabolic rate, etc.). A selected organism (here, in pink with a white square border) can be saved or put into an organism viewer to see its genome

and biological organisms, particularly bacteria. In addition, we implemented "best practices" for Avida-ED identified by Lark (2014), including implementing Avida-ED over the entire semester (long duration), having one of us (JS) carry out the actual implementation (high level of instructor expertise), scaffolding the implementation via a set of guided introductory exercises, and implementing Avida-ED in the lab in a way that was tied to the course curriculum.

We developed an Avida-ED Lab Book (Additional file 1) for fall 2014 consisting of an introduction to Avida$E D$, three introductory exercises targeting specific student misconceptions about evolutionary processes, and an independent research piece in which students used Avida-ED to answer a research question that they devised. The introductory exercises were drawn from a set individual exercises that had been developed previously and made available on the Avida-ED web site (http://avida-ed.msu.edu).

\section{The Avida-ED curriculum and its implementation in fall 2014}

\section{Educational context}

The curriculum was implemented in an Introductory Cell and Molecular Biology course (LB145) in a residential college on the campus of a major Research I University comprised mainly of students majoring in the natural sciences. Students typically take this introductory five-credit course in year 2 of their 4-year program (first-year chemistry is prerequisite). The course consists mainly of suburban and rural Michigan students, with approximately $10 \%$ of the students coming from traditionally underrepresented groups in the sciences. The students enter the class with a range of biology backgrounds and math preparedness. LB145 is taken by science majors (mainly Human Biology and Physiology), and includes many preprofessional students.

In the pilot implementation in spring 2014, there were 100 students in the lecture section, which was divided into five lab sections of 20 students each. The lecture was taught by the instructor (JS), while the labs were taught either by the instructor (one section) or one of two graduate teaching assistants, each assisted by two undergraduate learning assistants. Fall enrollment in the lecture section of LB145 was 39 students, divided again into two lab sections of 19-20 students each. Both lab sections were taught by the instructor, assisted by two undergraduate learning assistants in each case.

\section{The Avida-ED lab book}

The Avida-ED Lab Book (Additional file 1) represents the core component of the curriculum. The lab book begins with an introduction to Avida-ED, including Zimmer's (2005) Discover article, “Testing Darwin”, which describes digital organisms and how they are being used to study fundamental evolutionary processes (note: not included 
in Additional file 1), and a tutorial that takes students through the fundamentals of the use of the Avida-ED program. The introductory lesson is followed by three exercises (Exercises 1-3), each geared towards addressing particular learning goals with respect to evolutionary principles. These exercises not only serve to provide a scaffold for student learning about evolutionary principles, but also allow students to gain more familiarity with the Avida-ED platform. The Avida-ED Lab Book concludes with a description of and guide to an Independent Research Project, in which students worked in research teams to design and carry out their own experiments using Avida-ED.

Avida-ED Lab Book Exercise 1 and the Independent Investigation Exercise are modifications of exercises of the same name, while Exercise 2 is a modification of the exercise titled "Exploring Selection and Fitness", all originally produced by two of us (RTP and AL). Complete materials for these original lessons are available under the Curriculum link at http://avida-ed.msu.edu.

\section{Elements of implementation}

The full Avida-ED curriculum that was implemented in the fall 2014 Introductory Cell and Molecular Biology course consisted of classroom instruction in both the lecture hall and laboratory classroom, including background about digital evolution in general and Avida-ED in particular as an experimental model system. A summary of the implementation of Avida-ED in LB145 in fall 2014 is shown in Table 1. Activities primarily focused on the set of introductory exercises in The Avida-ED Lab Book that student teams completed, and an independent investigation in which student teams designed and carried out their own experiments using Avida-ED.

\section{Introduction to Avida-ED/introductory lesson}

Avida-ED was introduced in a lab session via a mini-lecture (approx. 15-20 min) followed by the assignment of the Introductory Lesson and Tutorial in The Avida-ED Lab Book. Students worked together in lab teams, which were chosen at the beginning of the semester using the Team-Maker program included in Purdue University's CATME package (http://catme.org). The Introductory Lesson and Tutorial was modified from the "Introduction to Digital Evolution Handout \& Tutorial" written by Johnson et al. (2009) for the Teach Engineering Curriculum for K12 Teachers (teachengineering.org). Students used this introductory activity to gain familiarity

Table 1 Timeline of activities and assessments associated with implementation of Avida-ED in LB145 S14 and LB145 F14

\begin{tabular}{|c|c|c|c|c|c|}
\hline \multicolumn{3}{|c|}{ Spring 2014} & \multicolumn{3}{|l|}{ Fall 2014} \\
\hline Week & Activities/assessments & Location & Week & Activities/assessments & Location \\
\hline Week 1 & & & Week 1 & & \\
\hline Week 2 & & & Week 2 & & \\
\hline Week 3 & & & Week 3 & $\begin{array}{l}\text { Lab book distributed; introduction to Avida-ED, } \\
\text { including lecture/video/demo and tutorial; } \\
\text { Abr question (pre-test) }\end{array}$ & Lab \\
\hline Week 4 & & & Week 4 & Introductory exercise quiz due; exercise 1 assigned & Lab \\
\hline Week 5 & & & Week 5 & $\begin{array}{l}\text { Exercise } 1 \text { group data explored; exercise } \\
2 \text { assigned; exercise } 2 \text { data explored; } \\
\text { Avida-ED/clam case tie-in }\end{array}$ & Lecture/lab \\
\hline Week 6 & & & Week 6 & $\begin{array}{l}\text { Exercise } 3 \text { assigned; guidelines and expectations for } \\
\text { proposal distributed }\end{array}$ & Lab \\
\hline Week 7 & & & Week 7 & $\begin{array}{l}\text { Demonstration of the proposal presentation; } \\
\text { rubric for proposal and presentation distributed }\end{array}$ & Lab \\
\hline Week 8 & $\begin{array}{l}\text { Introduction to Avida-ED, including lecture/ } \\
\text { video/demo and tutorial }\end{array}$ & Lecture & Week 8 & Student proposal presentations & Lab \\
\hline Week 9 & Tutorial assignment & Lecture & Week 9 & $\begin{array}{l}\text { Feedback on proposals; poster guidelines and } \\
\text { expectations distributed, with the scoring rubric }\end{array}$ & Lab \\
\hline Week 10 & Mini-lecture & Lecture & Week 10 & Avida-ED research & Lab \\
\hline Week 11 & & & Week 11 & Avida-ED research & Lab \\
\hline Week 12 & Exam questions & Lecture & Week 12 & Avida-ED research; Abr question (post-test) & Lab \\
\hline Week 13 & In class activity & Lecture & Week 13 & $\begin{array}{l}\text { Poster presentations in the BEACON center; } \\
\text { peer evaluation of posters }\end{array}$ & $\mathrm{N} / \mathrm{A}$ \\
\hline Week 14 & Activity debrief; $A b^{r}$ question & Lecture & Week 14 & & \\
\hline Week 15 & Avida-ED user's survey & Lecture & Week 15 & Avida-ED user's survey & Lecture \\
\hline Week 16 & Exam questions & Lecture & Week 16 & & \\
\hline
\end{tabular}


with Avida-ED and the concept of Avidians, the AvidaED program, and the user interface. They also read the article by Zimmer (2005), which introduced digital evolution research and helped students think about parallels between digital organisms and biological organisms.

\section{Avida-ED exercises 1-3}

Exercises 1-3 each were introduced briefly in a lab session (approx. $10 \mathrm{~min}$ ) and time was built into the lab schedule to allow students to work on these exercises with members of the teaching team present to provide direction and feedback. Students then worked individually outside of class on these three exercises, each of which required students to contribute their data to the pooled class data via Survey Monkey. The course instructor (JS) monitored and summarized the data for presentation and discussion in both the lecture section and the lab sections of the course. These pooled class data provided a powerful visual representation of the processes playing out in the Avida-ED program. Each exercise also had an associated ungraded quiz consisting of a set of free response-type questions pertaining to the exercise. Students were required to submit individual responses to these questions through the Desire2Learn course management system.

Avida-ED exercise 1: understanding the introduction of genetic variations by random mutation The primary learning goal for students in Exercise 1 was to be able to explain what it means to say that mutations occur at random. Students often carry the mistaken impression that evolution itself is random (Garvin-Doxas and Klymkowsky 2008; Mead and Scott 2010b). Exercise 1 addressed this point by showing that while mutations occur at random, natural selection itself is not random. We also explored the concept of a mutation rate, which provided the opportunity to discuss central tendencies and dispersion about a mean.

Students began Exercise 1 by replicating the "@ancestor" Avidian in the organism viewer with a $10 \%$ mutation rate applied. Students were asked to predict how many mutations they expected to see, and then recorded how many they actually observed. Students compared their initial results with those obtained by a classmate. Students were also asked to explore how their mutations were distributed as a function of position within the Avidian "genome" and to record the locations of each of the mutations that they observed. In this way, we illustrated the point that, in theory, every location in a genome is subject to mutation, even though not all mutations will survive to appear in later generations.

The students universally agreed that they expected to observe five mutations in the 50 positions of the Avidian genome when the organism replicated with a $10 \%$ mutation rate. However, the actual pooled class data shared with the students (Fig. 2a) clearly showed that not everyone observed five mutations in each run, as students often mistakenly expect; individual runs varied anywhere from one to 13 mutations. On the other hand, the mean number of mutations was 4.88 , very close to and not significantly different from the expected value of 5.00 (1-sample, 2-tailed $\mathrm{t}$ test; $\mathrm{t}=-0.5163, \mathrm{df}=80$; $\mathrm{p}=0.607$ ). (Note: All reported statistical analyses were performed in R version 3.2.3; R Core Team 2013) This allowed students to better see how randomness applies in practice. Students were also able to see visually that the 474 mutations they observed as a class were distributed across the Avidian genome, with each of the 50 positions in the Avidian genome experiencing at least one mutation event (pooled student class data shown in Fig. 2b).

Avida-ED exercise 2: exploring fitness, functions, and selection The primary learning goal for students in Exercise 2 was to be able to explain what it means to say that a trait has increased in frequency or has gone to fixation in a population. The difficulty of thinking in terms of populations instead of individuals is a recurring impediment to student understanding of evolutionary principles and processes (Bishop and Anderson 1990; Shtulman 2006; Gregory 2009), and one of the strongest features of Avida-ED is its ability to provide a dynamic visual representation of population-level processes. In Exercise 2, an individual Avidian is saved that can perform a particular function (the logic function, NOT). This organism is then separately put into environments either with or without the resource for that function (notose), which allows students to see how selection affects a trait when it provides a reproductive advantage.

Students began the exercise by evolving an Avidian population starting with "@ancestor", a simple self-replicator ancestor. After sufficient time passed (typically 100-200 updates), students located and isolated an individual Avidian that had evolved the ability to perform the logic function NOT. This individual was used to seed two subsequent independent Avida-ED runs (i.e., it was the ancestor in these runs). In the first of these two subsequent runs, there was no resource in the environment for the ability to perform NOT, which meant that there was no reward for performing NOT. After 2000 updates, students recorded the frequency in the population of individuals able to perform NOT (phenotype frequency). In the second run, the metabolic resource notose was included in the environment, which provided an energy reward to an organism if it performed NOT. Once again, after 2000 updates, students recorded the frequency in the population of individuals able to perform NOT. 

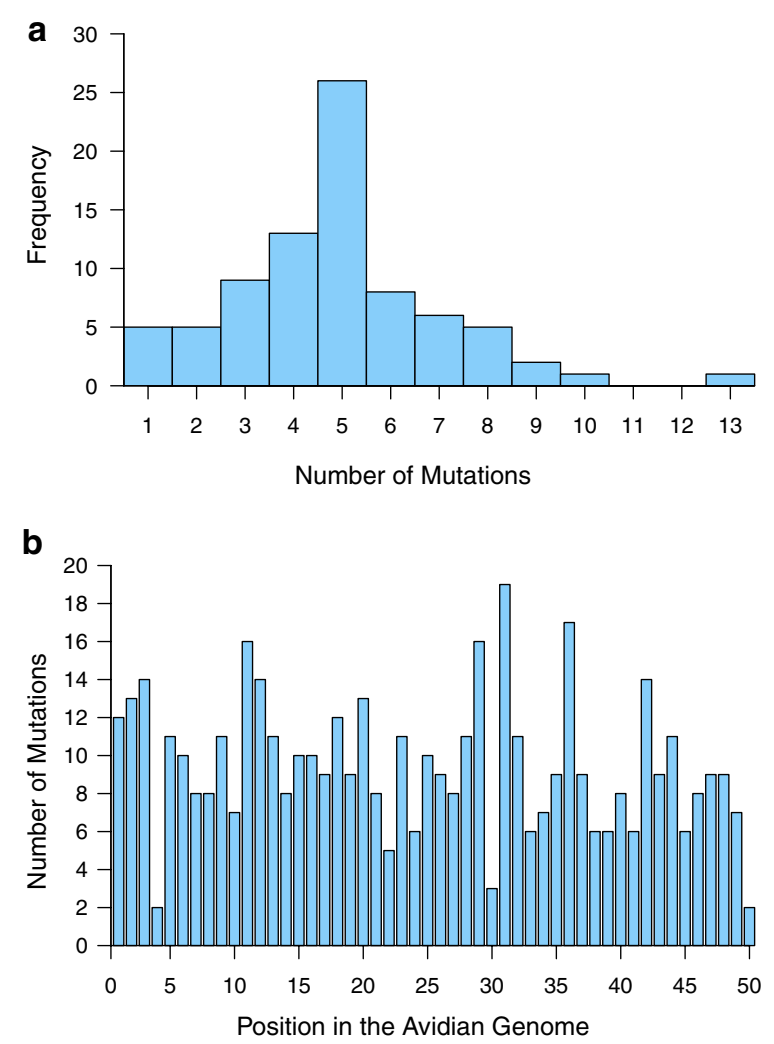

Fig. 2 Class data for Exercise 1 generated using Avida-ED. a Frequency of different \#'s of mutations per run. Data shown are for 90 responses from 30 students, and show that while the mean value for the runs is close to five, individual runs ranged from 1 to 13 .

b Distribution of mutations across the Avidian genome. The number of mutations is shown as a function of Avidian genome position, showing that all 50 positions in the genome are subject to mutation

Pooled data were collected (Fig. 3) and used in class to test the hypothesis that there was no difference in phenotype frequency (i.e., ability to perform NOT) between runs occurring under the two sets of conditions. Use of the actual class data allowed students to see that, as a whole, when the not phenotype was rewarded in the environment (notose was present), its frequency increased in the population over time. Notably, while most of the students obtained clear results in line with the aggregate data (28/32), some students had no data or ambiguous results. Particularly in these cases, being able to see and discuss the data obtained by peers, and to see the overall trend, was extremely valuable.

Avida-ED exercise 3-exploring mutations and selection: pre-adaptive or post-adaptive? One enduring misconception students hold is that evolutionary processes are forward looking and that events occur in anticipation of some future need (Mead and Scott 2010a). Thus, the

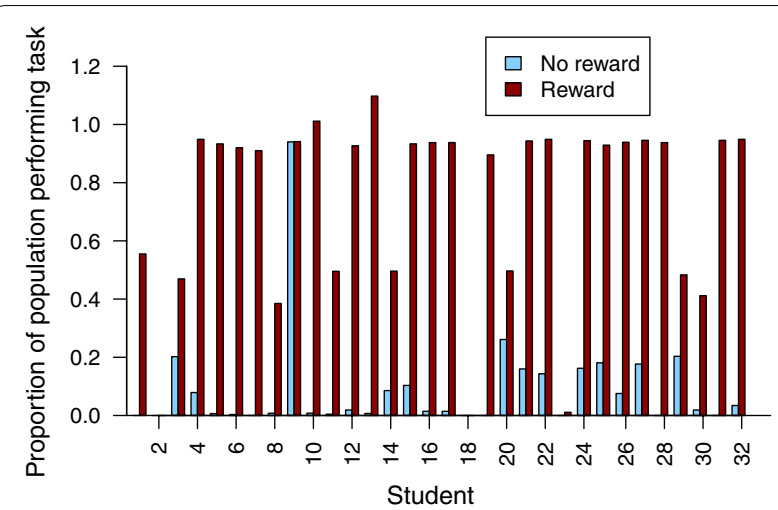

Fig. 3 Class data for Exercise 2 generated using Avida-ED. The frequency of NOT in the Avidian population after a set number of updates is plotted for each student's pair of trials, first with no reward for being able to perform NOT (light blue bars) and then with a reward for NOT (dark red bars). Overall, the data show that when NOT is rewarded, the trait goes to higher frequency and has a higher probability of persisting in an Avidian population. Importantly, not all students observed this outcome in their individual trials

primary learning objective in Exercise 3 is for students to demonstrate that they understand that mutations are random and do not occur simply because they are needed. Avida-ED provides a unique and direct way to test this evolutionary principle.

Students again begin the exercise by seeding a population with the @ancestor, making sure that all rewards are turned off (and thus, that the ability to perform a logic function does not confer a selective advantage). Students then begin their Avida-ED run, and stop the run when the first individual appears in the population that can perform the logic function NOT. Students then record the update at which this event occurred. Next, students repeat the exercise, but now set the initial environmental conditions such that the ability to perform NOT is rewarded (notose is present in the "medium"). Once again, the run is stopped when the first individual appears in the population that can perform NOT, and students record the update at which this event occurred.

When Exercise 3 was introduced with a clicker question, $65 \%$ of the students who responded indicated that they expected the mutation to occur sooner when it was rewarded. However, the actual aggregated data shown in class (Fig. 4) provided students with a different view. Almost all students had the function NOT occur in their populations more quickly under one condition or the other. However, the pooled data showed that there was no difference in the time to first appearance for a mutation in populations with or without reward (2-tailed binomial test, $\mathrm{p}=0.541$ ). 


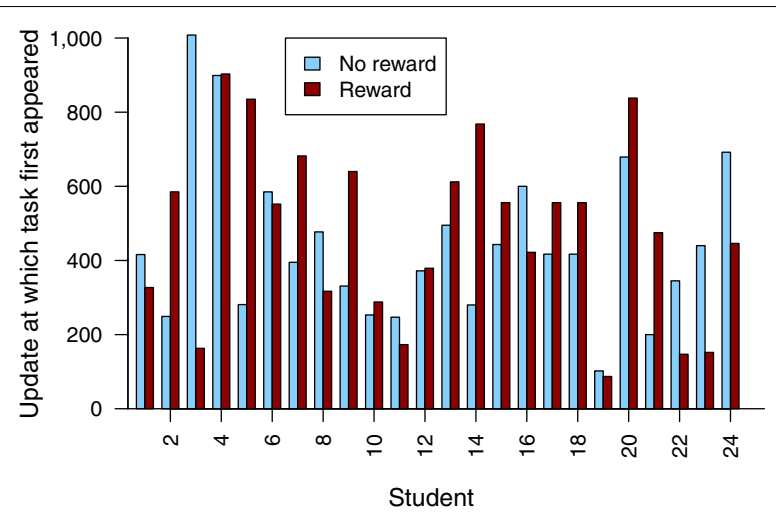

Fig. 4 Class data for Exercise 3 generated using Avida-ED. The number of updates to the first appearance of NOT is plotted for each student's pair of trials, first when no reward is present for being able to perform NOT (light blue bars) and then when there is a reward present for being able to perform NOT (dark red bars). In some instances, NOT appeared more quickly when there was a reward and, in other instances, NOT appeared more quickly when there wasn't a reward for it. Statistical analysis using a t test on the class data showed no difference in the time to first appearance of NOT in the presence or absence of reward

\section{Independent research investigation-experimental evolution} project with evolving digital organisms

Lark (2014) showed that students do better when they have substantial time and scaffolding to make sense of what they see in Avida-ED. After the students had completed the three preliminary exercises, and had time to explore the data they had generated as a class, the student teams were challenged to come up with a research question of their own to test using Avida-ED. This independent investigation component of the curriculum provided a way for students to do science and think like biologists, and we incorporated into these investigations all of the elements described by Thornton (1972) as adapted by Sundberg and Moncada (1994). Students were told that the purpose of the exercise was to engage in investigation, students consulted with teaching team members to formulate problems and investigatory procedures, students were provided ample time to repeat and/or modify experiments, and students prepared and presented both written and oral reports describing their work.

The Independent Investigations consisted of an initial exercise in which students worked within their research teams to write a research proposal that included a description of their research question, framed the question as a formal hypothesis to be tested, described the experimental design (including descriptions of relevant variables, what data they would collect, and how many replicates they would carry out), and stated a prediction of their expected outcomes if indeed their hypothesis was true. Teams then presented their proposals to the entire class with each team member explaining one part of it. The Guidelines and Expectations for the Proposal are included as Additional file 2, and a sample presentation slide set from one of the research teams is included as Additional file 3.

Student teams carried out their experiments in the teaching lab over the following 3-4 weeks. During this time, the teaching team met regularly with the student teams to check on progress, go over preliminary data, and suggest directions for next steps, much as would happen in an actual research laboratory. Students were also counseled with respect to how to organize their data within spreadsheets, and summarize their data in figures and tables that could be used in their poster presentations. How to organize the data collected during the experiments proved to be one of the biggest challenges faced by the teams, prompting one student to comment that, "I've never had so much data before!" A sample Excel file generated by a student team is included as Additional file 4.

Students put together their research posters using a set of guidelines and expectations that were distributed and discussed in the lab (Additional file 5). Preliminary drafts of the posters were discussed during a lab session and formal feedback provided for each group. During the Poster Presentation Session at the BEACON Center, each research team of four students was divided into two teams of two, and each team of two presented their team's poster and fielded questions about their research. A sample poster from one of the research teams is included as Additional file 6.

The Avida-ED Independent Research Project allowed students to engage in authentic science practices ("do science"), thus addressing a number of our science process skills objectives. Students worked in teams to brainstorm ideas, ask questions, develop hypotheses, design experimental plans, conduct experiments and collect data, analyze data, and write results for presentation to peers and experts. Students were given time to think about their projects and feedback was provided by the teaching team both informally in conversations in the planning stage and formally in response to their proposal presentations. All of these elements provided practice for our students doing the kinds of things that scientists do when they conduct research.

\section{Initial assessment of student learning} Preliminary assessment of student learning using Avida-ED In both spring semester 2014 and fall semester 2014, students using Avida-ED were given an assessment item asking them to "Explain how a microbial population evolves resistance to the effects of an antibiotic". In spring 2014, a quasi-experimental protocol was employed and 


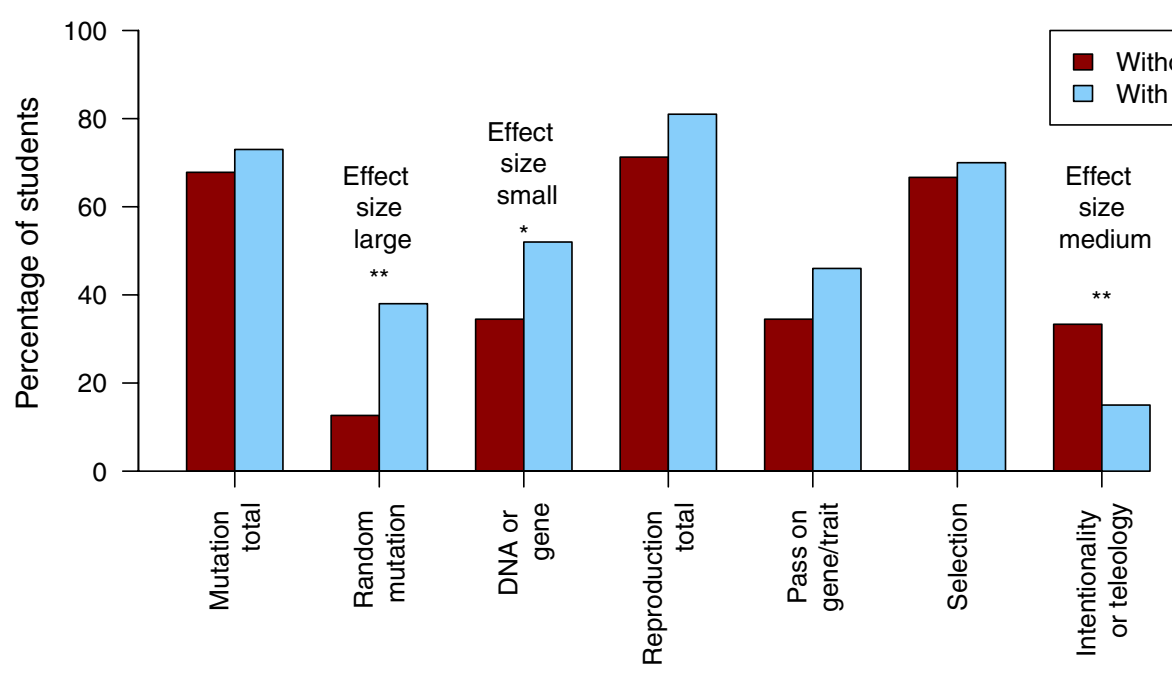

Answer contains term

Fig. 5 Concepts present in student explanations of the evolution of antibiotic resistance in a microbial population. Post-instruction, students in spring 2014 in both the LB145 classroom that used Avida-ED $(n=100)$ and the LB145 classroom that did not use Avida-ED $(n=87)$ were asked, "Explain how a microbial population evolves resistance to the effects of an antibiotic". Responses were assessed for the presence of concepts identified using content analysis. Significance of differences within categories was assessed using Chi squared tests. ${ }^{* *} p<0.01 ;{ }^{*} p<0.05$. Effect sizes reported as odds-ratios per Maher et al. (2013)

responses from students in the Avida-ED classroom $(\mathrm{n}=100)$ were compared to responses from students not using Avida-ED (control; $\mathrm{n}=87$ ). We collected all data following Michigan State University Institutional Review Board guidelines, IRB \#i040365. Preliminary analysis of these data, using content analysis (Weber 1990) to identify emerging themes and the frequency of their occurrence in the student responses, indicated that the students in the Avida-ED classroom more frequently mentioned the key concepts "random mutation" ( $t$ test; $\mathrm{p}<0.01$ ) and "DNA or gene" ( $t$ test; $\mathrm{p}<0.05$ ) in response to the prompt than did the students in the classroom that did not use Avida-ED (Fig. 5). The Avida-ED students also used the naïve ideas of intentionality and teleology less often in their responses than did the students in the non-Avida-ED class (t test; $\mathrm{p}<0.01$ ).

In fall semester 2014, we employed a pre-/post-test design using the same question for the Avida-ED classroom $(n=39)$. In this case, the resulting text responses were used as an input file for the EvoGrader application (Moharreri et al. 2014). A higher proportion of the students displayed "Pure Scientific" reasoning in the posttest (Fig. 6a) and students in the post-tests strengthened connections between core concepts and mentioned fewer naïve ideas (Fig. 6b).

\section{Students affective response to the Avida-ED curriculum}

Student affective responses to the Avida-ED curricular materials were assessed at the end of the semester in both spring 2014 and fall 2014 using an Avida-ED User's Survey (Additional file 7). Overall, student response to the Avida-ED implementation was better in fall 2014 (full implementation) than it was in spring 2014 pilot implementation. Evidence in support of this claim was obtained from the item in the User's Survey asking students which category best describes their overall enjoyment of AvidaED (Fig. 7). In fall 2014, the percentage of students who "loved" Avida-ED increased by approximately tenfold, while the number who "hated it" decreased to zero. These results are consistent with the findings of Lark (2014), who found a positive correlation between the use of best practices in the implementation of Avida-ED and positive student affective responses.

\section{Discussion}

In this paper, we have described the development of an Avida-ED course curriculum that was implemented in an Introductory Cell and Molecular Biology course (LB145) taught by one of us (JS) over two semesters (spring 2014 and fall 2014). The pilot implementation in spring 2014 occurred mainly in the lecture hall and required students to work on assignments outside of class, without a lot of guidance or support. This is likely the explanation for the dramatic increase in affective response from spring 2014 to fall 2014 (Fig. 6), when the curriculum was better constructed to incorporate many of the best practices for Avida-ED implementation identified by Lark (2014). 


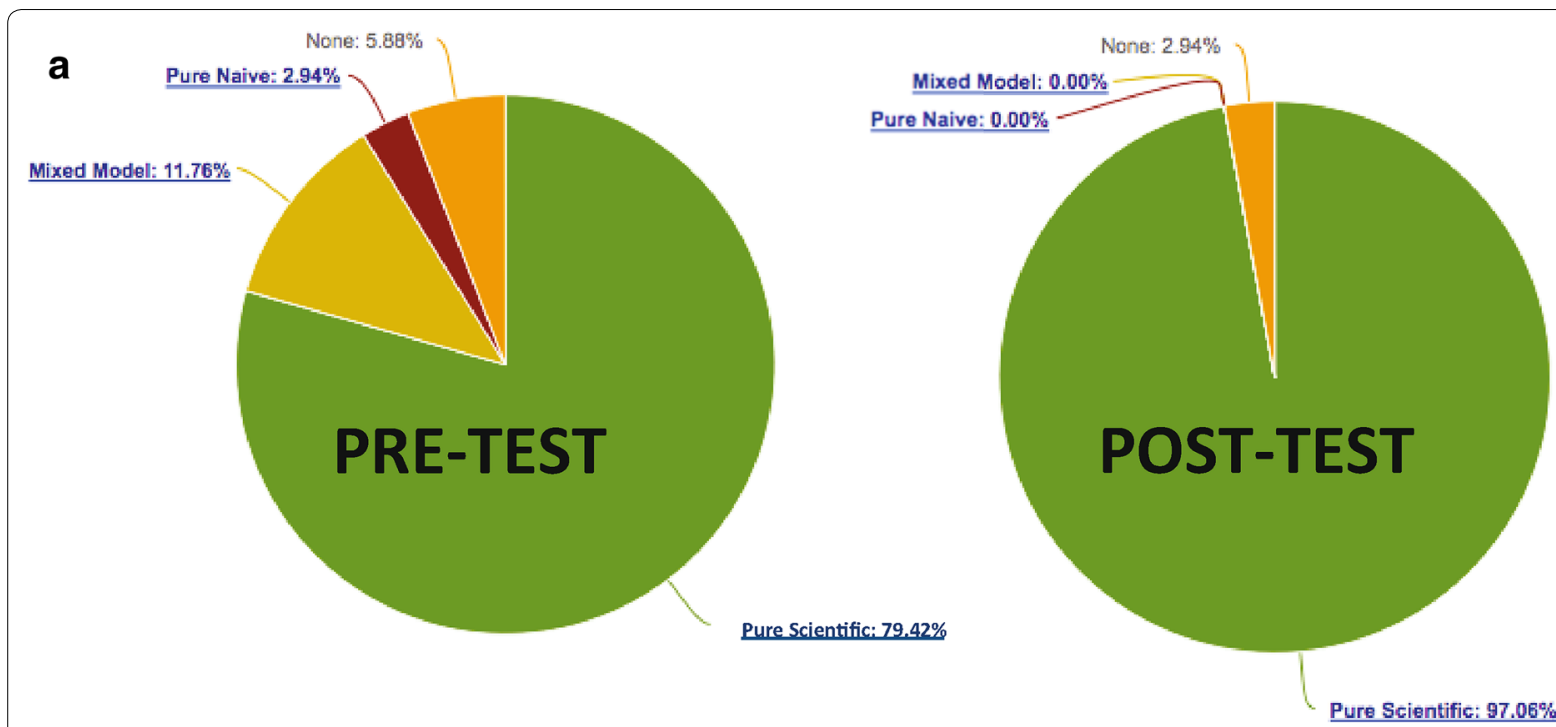

b PRE-TEST

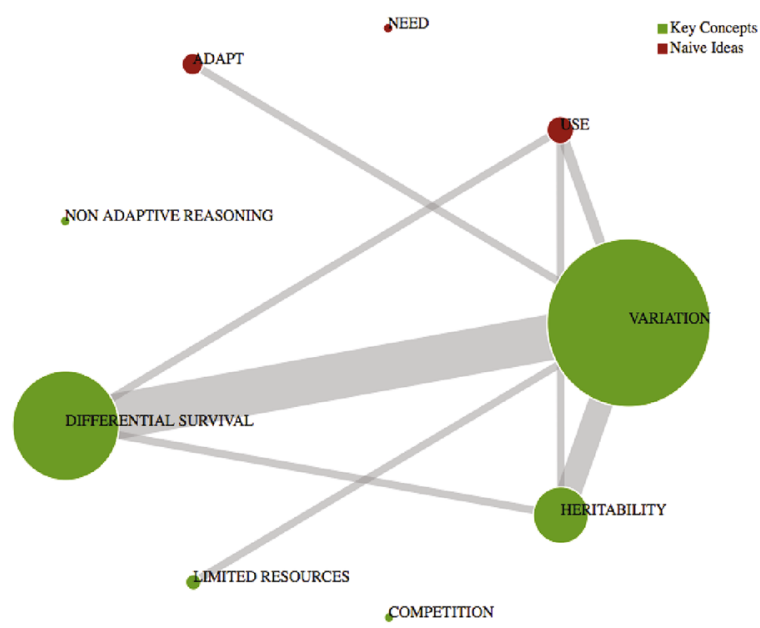

POST-TEST

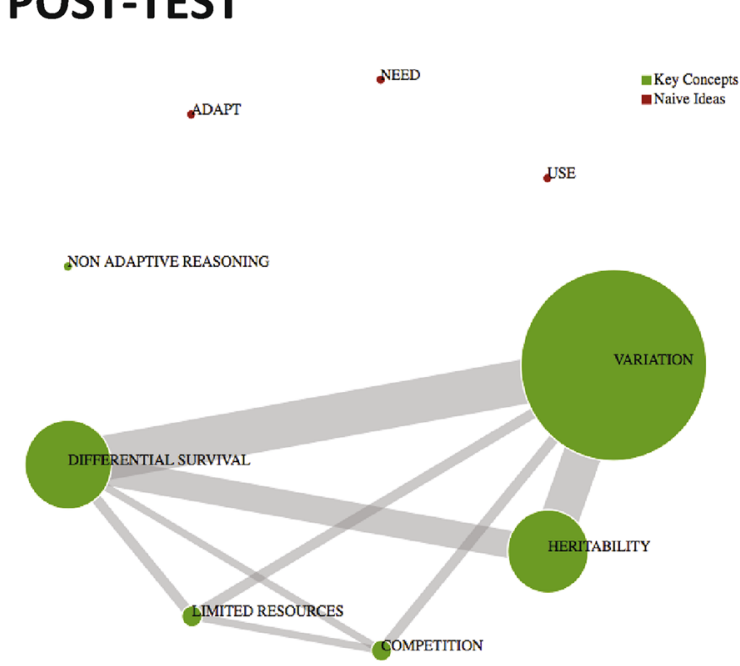

Fig. 6 EvoGrader analysis of student explanations of the evolution of antibiotic resistance in a microbial population. a Pie charts show percentage of student whose responses $(n=39)$ displayed "Pure Scientific", "Pure Naïve", or a "Mixed Model” of reasoning. b Responses were scored for the frequency of the appearance of core concepts and naïve concepts, as well as the number of connections made between them

Full implementation of the Avida-ED curriculum as described here may not be possible for others in their own courses, especially if learning objectives are different from our own. However, we encourage instructors to adapt and use whichever parts of the curriculum they might find useful, depending on the learning outcomes desired. The different parts of the curriculum can be adapted for use in isolation, and we encourage those who adopt Avida-ED to start with the Lab Book included here and create something new for their own use. This is the intent of the Creative Commons license under which the Lab Book was produced.

\section{Possible curricular modifications and extensions}

One modification that practitioners might find useful would be to reverse the order of Exercises 2 and 3. By doing Exercise 3 first, students will have already evolved an Avidian that can perform the logic function not, which is required for Exercise 2, prior to the beginning of this exercise. The original ordering of Exercises 2 and 3 will be fine for those with adequate time, but the modification will save time for those who need it.

Also, a number of additional curricular materials are available at the Avida-ED website (http://avida-ed.msu. edu), including three additional Avida-ED exercises 


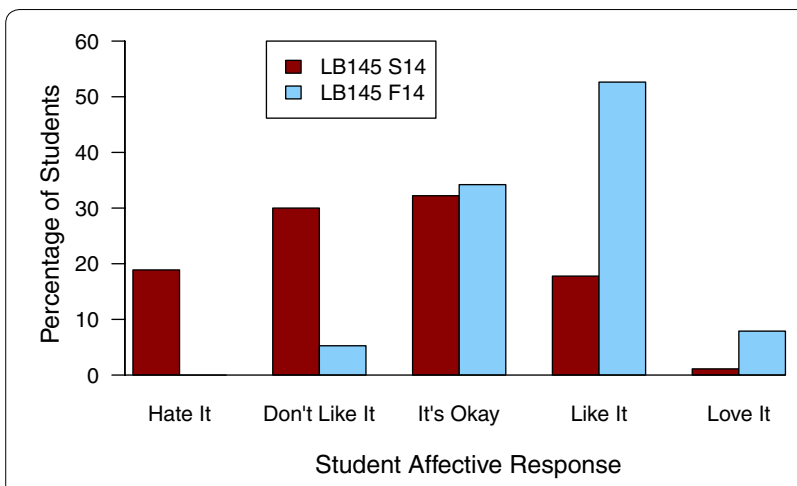

Fig. 7 Results of Avida-ED user's survey of student enjoyment of Avida-ED. Students in both spring 2014 and fall 2014 were asked to indicate which of five categories best described their overall enjoyment of Avida-ED. Values shown on the $y$-axis are the percentage of responses (spring 2014, $n=90$; fall 2014, $n=38$ )

previously developed in conjunction with the Avida-ED Curriculum Development Group: "Exploring the Effects of Mutation Rate on Individuals", a lesson based on the Fukushima nuclear disaster (Lark et al. 2014); "From Genotype to Phenotype: Understanding the Introduction of Phenotypic Variations"; and "Evolving TCE Biodegraders". Student handouts are provided at the website for each of these exercises, as are web links to more complete curricular materials.

\section{Conclusions and future directions}

Evolution presents a challenging set of ideas and concepts for students to master, with students regularly incorporating naïve and scientific concepts simultaneously (Nehm and Ha 2011). Our data indicate that using Avida-ED helps students understand the role of mutation, and may minimize student use of naïve concepts. Thus, Avida-ED appears to hold great promise as a tool that students can use to learn evolutionary principles and to carry out scientific investigations of evolutionary processes.

\section{Additional files}

Additional file 1. The Avida-ED lab book. Pdf of the lab book used in fall 2014 (minus the Zimmer Discover article).

Additional file 2. Avida-ED independent research project proposal guidelines and expectations.

Additional file 3. Student Work showing the slide set presented by one student team during their oral proposal presentation.

Additional file 4. Excel file generated by the same student team during their Avida-ED independent investigation.

Additional file 5. Avida-ED poster guidelines and expectations.

Additional file 6. Sample student poster that was presented at the BEACON Center in Nov. 2014.

Additional file 7. Avida-ED user's survey.

\section{Authors' contributions}

JJS, WRJ, AML, LSM and RTP developed curricular materials and implemented them in the classroom. All authors collected and analyzed data. MJW did the statistical analyses. JJS and RTP wrote the manuscript. All authors provided comments on the final manuscript. All authors read and approved the final manuscript.

\section{Author details}

${ }^{1}$ Michigan State University, Lyman Briggs College, 919 E. Shaw Lane, Room E35, East Lansing, Ml 48825-3804, USA. ${ }^{2}$ Department of Entomology, Michigan State University, 288 Farm Lane, Room 243, East Lansing, Ml 48824, USA. ${ }_{3}^{3}$ Department of Integrative Biology, Michigan State University, 288 Farm Lane, Room 203, East Lansing, MI 48824-1115, USA. ${ }^{4}$ BEACON Center for the Study of Evolution in Action, Michigan State University, 567 Wilson Road-BPS Room 1441, East Lansing, MI 48824, USA. ${ }^{5}$ Program in Ecology, Evolutionary Biology and Behavior, Michigan State University, East Lansing, MI 48824, USA. ${ }^{6}$ Department of Teacher Education, Michigan State University, Erickson Hall, 620 Farm Lane, Room 313, East Lansing, MI 48824, USA. ${ }^{7}$ Department of Cognitive and Learning Sciences, Michigan Technological University, 1400 Townsend Drive, Houghton, MI 49931, USA. ${ }^{8}$ Department of Philosophy, Michigan State University, 503 S. Kedzie Hall, East Lansing, MI 48824, USA. ${ }^{9}$ Department of Computer Science \& Engineering, Michigan State University, 428 South Shaw Lane, East Lansing, Ml 48824, USA.

\section{Acknowledgements}

We thank all of the LB145 students in spring 2014 and fall 2014 for using Avida-ED as a part of their learning experience. Special thanks go to the team members of the research team "Black Mamba", who contributed their Proposal Presentation slide set, their data file, and a copy of their final poster for inclusion in this paper. We also thank Dr. Peter J. T. White and his LB145 students for serving as an Avida-ED "control" group in spring semester 2014. This material is based in part upon work supported by grants from the Howard Hughes Medical Institute through the Science Education Program to Michigan State University, Pennock (Co-PI), Mead and Smith (Senior Personnel), and from the National Science Foundation IUSE No. 1432563, Pennock (PI), Mead, and Smith (Co-PIs). Any opinions, findings, and conclusions or recommendations expressed in this material are those of the author(s) and do not necessarily reflect the views of the funding agency. Datasets and analysis scripts are available at the Dryad Digital Repository (doi:10.5061/dryad.7t457 Available upon acceptance).

\section{Competing interests}

The authors declare that they have no competing interests.

Received: 9 February 2016 Accepted: 13 July 2016

Published online: 29 July 2016

\section{References}

Abraham J, Meir E, Perry J, Herron J, Maruca S, Stal D. Addressing undergraduate student misconceptions about natural selection with an interactive simulated laboratory. Evol Educ Outreach. 2009;2:393-404.

Alters BJ, Nelson CE. Perspective: teaching evolution in higher education. Evolution. 2002;56:1891-901.

Bishop B, Anderson CW. Student conceptions of natural selection and its role in evolution. J Res Sci Teach. 1990;27:41-57.

Clune J, Goldsby HJ, Ofria C, Pennock RT. Selective pressures for accurate altruism targeting: evidence from digital evolution for difficult-to-test aspects of inclusive fitness theory. Proc Biol Sci. 2011;278:666-74. doi:10.1098/ rspb.2010.1557.

Elena S, Sanjuan R. The effect of genetic robustness on evolvability in digital organisms. BMC Evol Biol. 2008;8:284.

Elwood J, Klenowski V. Creating communities of shared practice: the challenges of assessment use in learning and teaching. Assess Eval High Educ. 2002;27:243-56. doi:10.1080/02602930220138606.

Garvin-Doxas K, Klymkowsky MW. Understanding randomness and its impact on student learning: lessons learned from building the biology concept inventory (BCI). CBE Life Sci Educ. 2008;7:227-33. doi:10.1187/ cbe.07-08-0063. 
Goldsmith DW. The great clade race: presenting cladistic thinking to biology majors \& general science students. Am Biol Teach. 2003;2003:679-83.

Gregory TR. Understanding natural selection: essential concepts and common misconceptions. Evol Educ Outreach. 2009:2:156-75.

Johnson WR. Evolution in action in the classroom: Engaging students in scientific practices to develop a conceptual understanding of natural selection (Master's thesis). ProQuest Dissertations and Theses database. (UMI No. 1517061). 2012.

Johnson W, Pennock RT, Mead L. Studying evolution with digital organisms activity —introduction to digital evolution handout \& tutorial. Teach Engineering Curriculum for K12 Teachers. 2009. http://teachengineering. org. Accessed 28 Dec 2015.

Lark AM. Teaching and learning with digital evolution: factors influencing implementation and student outcomes (Doctoral dissertation). ProQuest Dissertations and Theses database. (UMI No. 3645332). 2014.

Lark A, Richmond G, Pennock RT. Modeling evolution in the classroom: the case of Fukushima's mutant butterflies. Am Biol Teach. 2014;76:450-4. doi:10.1525/abt.2014.76.7.6.

Lenski RL, Ofria C, Pennock RT. The evolutionary origin of complex features. Nature. 2003;423:139-45.

Luckie DL, Smith JJ, Cheruvelil KS, Fata-Hartley C, Murphy CA, Urquhart GR. The "Anti- Cookbook Laboratory": converting "Canned" introductory biology laboratories to multi-week independent investigations. Proc Assoc Biol Lab Educ. 2013;34:196-213.

Maher JM, Markey JC, Ebert-May D. The other half of the story: effect size analysis in quantitative research. CBE_Life Sci Educ. 2013;12:345-51. doi:10.1187/cbe.13-04-0082.

Mead LS, Scott EC. Problem concepts in evolution part I: purpose and design. Evol Educ Outreach. 2010a;3:78-81.

Mead LS, Scott EC. Problem concepts in evolution part II: cause and chance. Evol Educ Outreach. 2010b;3:261-4.

Misevic D, Ofria C, Lenski RE. Sexual reproduction reshapes the genetic architecture of digital organisms. Proc Biol Sci. 2006;273:457-64. doi:10.1098/ rspb.2005.3338.

Moharreri K, Ha M, Nehm RH. EvoGrader: an online formative assessment too for automatically evaluating written evolutionary explanations. Evol Educ Outreach. 2014;7:15.
Nehm RH, Ha M. Item feature effects in evolution assessment. J Res Sci Teach. 2011;48:237-56.

Nelson C. Why don't undergraduates really "get" evolution? What can faculty do? In: Brem SK, Rosengren KS, Evans EM, Sinatra GM, editors. Evolution challenges: integrating research and practice in teaching and learning about evolution. Oxford: Oxford University Press; 2012.

Ofria C, Wilke CO. Avida: a software platform for research in computational evolutionary biology. J Artif Life. 2004;10:191-229.

Ofria C, Adami C, Collier TC. Selective pressures on genomes in molecular evolution. J Theor Biol. 2003;222:477-83.

Pennock RT. Learning evolution and the nature of science using evolutionary computing and artificial life. McGill J Educ. 2007a;42:211-24.

Pennock RT. Models, simulations, instantiations and evidence: the case of digital evolution. J Exp Theor Artif Intell. 2007b;19:29-42.

$R$ Core Team. R: a language and environment for statistical computing. Vienna: R Foundation for Statistical Computing. 2013. (http://www.R-project. $\operatorname{org} /$ ).

Shtulman A. Qualitative differences between naïve and scientific theories of evolution. Cogn Psychol. 2006;52:170-94.

Speth EB, Long T, Pennock RT, Ebert-May D. Using Avida-ED for teaching and learning about evolution in undergraduate introductory biology courses. Evol Educ Outreach. 2009:2:415-28.

Sundberg MD, Moncada GJ. Creating effective investigative laboratories for undergraduates. Bioscience. 1994;44:698-704.

Thornton J. The laboratory: a place to investigate. Commission on Undergraduate Education in the Biological Sciences. Washington DC: American Institute of Biological Sciences. p. 158. 1972.

Weber RP. Basic content analysis. 2nd ed. Newbury Park: Sage; 1990.

White PJT, Heidemann MJ, Loh M, Smith JJ. Integrative cases for teaching evolution. Evol Educ Outreach. 2013;6:1-7.

Zaman L, Meyer JR, Devangam S, Bryson DM, Lenski RE, Ofria C. Coevolution drives the emergence of complex traits and promotes evolvability. PLoS Biol. 2014:12:12. doi:10.1371/journal.pbio.1002023.

Zimmer C. Testing Darwin. Discover. 2005;26:28-35.

\section{Submit your manuscript to a SpringerOpen ${ }^{\circ}$ journal and benefit from:}

- Convenient online submission

- Rigorous peer review

- Immediate publication on acceptance

- Open access: articles freely available online

- High visibility within the field

- Retaining the copyright to your article

Submit your next manuscript at $\boldsymbol{\nabla}$ springeropen.com 